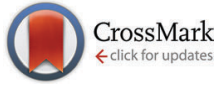

Cite this: Phys. Chem. Chem. Phys., 2015, 17, 19806

Received 31st March 2015 Accepted 23rd June 2015

DOI: $10.1039 / \mathrm{c} 5 \mathrm{cp} 01879 \mathrm{j}$

www.rsc.org/pccp

\section{Targets, ripples and spirals in a precipitation system with anomalous dispersion $\dagger$}

\begin{abstract}
Mahmoud M. Ayass, ${ }^{a}$ Istvan Lagzi ${ }^{\mathrm{b}}$ and Mazen Al-Ghoul*a
We present a novel reaction-diffusion system that exhibits three-dimensional superdiffusive traveling waves without utilizing any external forces. These waves include single circular targets, spirals, and ripples as well as phase-like waves. The system is based on the interplay of the precipitation reaction of mercuric iodide in a gel medium, its polymorphic transformation to a different crystalline form, and its redissolution in excess iodide. A phase diagram is constructed as a function of the initial concentrations of the reagents. The spatiotemporal evolution of these waves is thoroughly analyzed and seems to be a consequence of an anomalous dispersion relationship. Pattern selection and wavelengths of propagating waves are found to depend on initial concentrations of the reactants. The breakup of the waves is also investigated. While the breakdown of ripples and spirals is shown to be a consequence of a Doppler-like instability in conjunction with anomalous dispersion, the targets undergo a boundary defect-mediated breakup.
\end{abstract}

\section{Introduction}

Self-organized patterns in reaction-diffusion (RD) systems have been the subject of a tremendous amount of investigations by researchers in chemistry, ${ }^{1,2}$ physics ${ }^{3,4}$ and biology ${ }^{5,6}$ due to their high relevance in these fields. For chemical systems in particular, self-organization includes precipitation patterns in gel media, ${ }^{7-9}$ self-oscillating reactions in the homogeneous liquid phase, ${ }^{10}$ traveling waves ${ }^{11}$ and Turing patterns. ${ }^{12,13}$ Common ingredients in these examples are oscillatory reactions or excitable media. The Belousov-Zhabotinsky (BZ) system, ${ }^{11}$ reported in Nature in the year 1970, is the classical and most studied example that has provided the foundation for many recent self-oscillating systems that produce self-organizing patterns in homogeneous RD systems. Furthermore, heterogeneous auto-catalytic chemical reactions exhibiting various patterns have also been encountered in $\mathrm{RD}$ systems and have also been intensively studied. ${ }^{14}$ One prominent reaction of this type is the catalytic oxidation of $\mathrm{CO}$ on platinum crystal planes. ${ }^{15}$

In both, homogeneous and heterogeneous RD systems, the formation of many interesting patterns is the result of travelling waves which could take the shape of a spiral or a circular target pattern. ${ }^{16}$ The rotation of these spirals on a surface causes

\footnotetext{
${ }^{a}$ Department of Chemistry, American University of Beirut, P.O. Box 11-0236, Riad El-Solh 1107 2020, Beirut, Lebanon. E-mail: mazen.ghoul@aub.edu.lb; Fax: +961-1-365217; Tel: +961-1-350000 ext. 3970

${ }^{b}$ Department of Physics, Budapest University of Technology and Economics, Budapest H-1521, Hungary

$\dagger$ Electronic supplementary information (ESI) available: Two videos are provided displaying the propagation of the spiral and target patterns. An extra file is provided explaining the contents of each video. See DOI: $10.1039 / \mathrm{c} 5 \mathrm{cp} 01879 \mathrm{j}$
}

deformations in the medium of reaction. These deformations may further lead to the breakup of the spiral wave giving rise to complex spatiotemporal patterns ${ }^{4}$ and chemical turbulence. All of the aforementioned systems produce, under no advection, propagating fronts with constant velocities, ${ }^{17,18}$ or velocities in conformity with normal diffusive profile leading to a linear relation between the mean square spread of particles with time. Although recent experiments have confirmed the occurrence of superdiffusive chemical waves, such anomalous behavior is exhibited when the system is subjected to external modulations that enhance diffusion. ${ }^{21-25}$ Recently, the authors reported the propagation and interaction of three-dimensional superdiffusive target patterns in the absence of external forces in a novel heterogeneous system, namely the mercuric iodide precipitation system. ${ }^{19}$ In this sequel work, we present a full study of the aforementioned mercuric iodide system. A multitude of wave patterns, including targets (single circular wave), spirals, ripples as well as phase-like waves, are obtained. The propagation dynamics of all these waves is also shown to be superdiffusive and their breakup leads to defectmediated chemical turbulence. Many of the observed phenomena can be shown to be a result of anomalous dispersion relations, whereby the velocity of the pulse $c(T)$ increases by decreasing period $T$ (i.e. $\mathrm{d} c / \mathrm{d} T<0$ ), with striking similarities to those found in a special class of the $\mathrm{BZ}$ reaction. ${ }^{20,21}$

This paper is organized as follows. In Section 2, we describe the experimental procedure and setup. In Section 3, we report and discuss our results, which include the detailed chemistry (Section 3.1), the phase diagram of the various patterns observed and their dynamics in addition to their dispersion relations (Section 3.2), and the last part includes the discussion of the wave breakup mechanisms (Section 3.3). 


\section{Experimental}

The materials used in our experiments constitute of mercuric chloride (Fischer Chemical), potassium iodide (Merck) and agar gel (Bacto). We carried out all the experiments in gel media. We prepared stock solutions, using double distilled water, of both mercuric chloride $\left(\mathrm{HgCl}_{2}\right)$ and potassium iodide (KI) according to the required concentrations. We dissolved the agar powder (1\% volume) in a volume of $\mathrm{HgCl}_{2}$ solution (inner electrolyte) by using a magnetic stirring rod on a stirring/hot plate. We maintained the temperature of the mixture within a range of 80 $90{ }^{\circ} \mathrm{C}$. To ensure that no errors in the concentration occur we covered the beakers while stirring and made sure that the solutions never reach the boiling point. The solutions continue to mix until they become clear and no gel particles remain floating. When the solution mixtures were complete, we poured them into small Petri dishes ( $47.0 \mathrm{~mm}$ diameter). About $10 \mathrm{~mL}$ of each solution is transferred to each Petri dish, which forms a gel of about $7 \mathrm{~mm}$ in thickness. We then covered the plates and placed them in a chamber equipped with a thermostat that maintains the temperature at $20 \pm 0.2^{\circ} \mathrm{C}$. The gels were left for 2 hours for the completion of their gelation and aging processes.

Next, we proceeded with the performance of the reaction by pouring the previously prepared KI solutions (outer electrolyte) onto the surface of the solidified gel (initialization step). The temperature of the medium was kept at $20{ }^{\circ} \mathrm{C}$ during the reaction. We monitored the reactions under a high-resolution digital camera (Cannon EOS 450D) connected to an iMac. The mode that we used for our analysis took a clear focused shot of the plates and the software was set to take a snapshot every 5 seconds. The reactions proceeded for around 15-30 minutes each; therefore with our settings we produced around 200-400 snapshots of the reactions. Combining these frames shows us a clear time evolution of the traveling waves in our system.

\section{Results and discussion}

\subsection{Detailed chemistry}

The observations we report include the coexistence of moving precipitation patterns (as in the Liesegang phenomenon) with traveling waves (as in excitable media), perpendicular to the diffusion flux vector of the invading electrolyte inside the precipitate. In our system, $\mathrm{HgCl}_{2}$ (inner electrolyte) is evenly distributed in the agar gel, and then the KI (outer electrolyte) is poured on to the gel surface, which diffuses into the gel forming $\mathrm{HgI}_{2}$ precipitates. Specifically, the formation of $\mathrm{HgI}_{2}$ in gel media ${ }^{22}$ in the wake of a diffusion front results in three different polymorphs: orange (tetragonal), $\beta$-yellow-HgI ${ }_{2}$ (orthorhombic) and $\alpha$-red-HgI $\mathrm{I}_{2}$ (tetragonal). ${ }^{23}$ The orange and yellow forms are the kinetically favored polymorphs, thus appearing at early stages of diffusion, but they are not thermodynamically stable. With the slightest touch these polymorphs would readily transform, by alteration of the crystal structure, to the more stable and thermodynamically favored the red form of the compound..$^{24,25}$ Furthermore, in the presence of excess potassium iodide solution (outer electrolyte), both $\alpha$ - and $\beta$ - $\mathrm{HgI}_{2}$ precipitates rapidly dissolve forming the $\mathrm{K}_{2} \mathrm{HgI}_{4}$ complex. ${ }^{26}$ The aforementioned precipitation process, polymorphic transformation and the complex formation are summarized according to the following reaction scheme:

$$
\begin{gathered}
\mathrm{Hg}^{2+}(\mathrm{aq})+2 \mathrm{I}^{-}(\mathrm{aq}) \rightleftharpoons \beta-\mathrm{HgI}_{2}(\mathrm{~s}) \\
\beta-\mathrm{HgI}_{2}(\mathrm{~s}) \rightarrow \alpha-\mathrm{HgI}_{2}(\mathrm{~s}) \\
\alpha-\mathrm{HgI}_{2}(\mathrm{~s})+2 \mathrm{I}^{-}(\mathrm{aq}) \rightleftharpoons\left[\mathrm{HgI}_{4}\right]^{2-}(\mathrm{aq})
\end{gathered}
$$

Since the only contact between the two electrolytes is the surface of the gel, a thin layer of the precipitates $(\sim 70-150 \mu \mathrm{m})$ forms at the interface immediately after contact between the inner and outer electrolytes. As diffusion continues, the excess outer electrolyte following the front reacts with the precipitate to form the complex $\mathrm{K}_{2} \mathrm{HgI}_{4}$ (redissolution) and the whole layer appears like a front propagating downward. On the other hand, while monitoring the surface of the precipitate layer from a top view parallel to the surface of the gel, we observe distinct self-organized patterns forming inside the whole width of the thin precipitation disk. Fig. 1 represents the three different self-organized patterns we observe in our system.

A close inspection of the snapshots taken of the pattern evolution reveals that the tiny bubbles formed in the hot gel while solidifying serve as nucleation sites for all propagating patterns. Moreover, the glass boundary of our reactor acts also as a significant nucleation site, which produces rather irregular

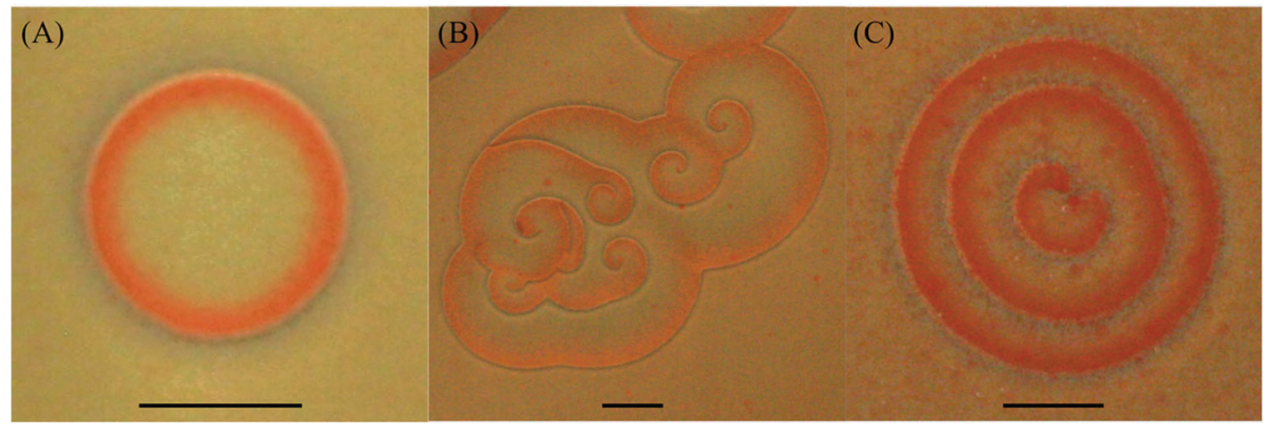

Fig. 1 Three different representative self-organized patterns forming in $\mathrm{Hgl}_{2}$ precipitate (top view). Initial conditions, (A) target, $\left[\mathrm{Hg}{ }^{2+}\right]_{0}=0.22 \mathrm{M}$, $\left[\mathrm{I}^{-}\right]_{0}=1.0 \mathrm{M}, 350 \mathrm{~s}$ after initialization; (B) spirals, $\left[\mathrm{Hg}^{2+}\right]_{0}=0.24 \mathrm{M},\left[^{-}\right]_{0}=5.0 \mathrm{M}, 460 \mathrm{~s}$ after initialization; (C) double spirals or ripples, $\left[\mathrm{Hg}^{2+}\right]_{0}=0.22 \mathrm{M}$ $\left[\mathrm{I}^{-}\right]_{0}=4.0 \mathrm{M}, 420 \mathrm{~s}$ after initialization. The scale bar represents $0.25 \mathrm{~cm}$. 
shaped patterns. All the measurements and descriptions reported correspond to patterns forming at the center of our reactor without any boundary interference.

The spatial evolution of the fronts over a particular region is initiated on a steady yellow background of $\beta-\mathrm{HgI}_{2}$. The front itself is a transformation wave that causes an increase in red $\alpha-\mathrm{HgI}_{2}$ and the subsequent decrease in $\beta-\mathrm{HgI}_{2}$. After the wave passes through this particular region, $\alpha-\mathrm{HgI}_{2}$ drops slowly and transforms back to a quasi-steady region equivalent to the composition of the background $\beta-\mathrm{HgI}_{2}$. We also notice, as the front travels, that it has a colorless outline which is presumed to be the complex $\left[\mathrm{HgI}_{4}\right]^{2-}$ appearing to be an intermediate product of the polymorphic transformation reaction between $\alpha-\mathrm{HgI}_{2}$, composing the front, and $\beta-\mathrm{HgI}_{2}$, composing the background (see ESI $\dagger$ ). The spatial description given above is similar to what is observed in the alteration of red and blue colors appearing in the $\mathrm{BZ}$ reaction, ${ }^{27}$ yet it is very unique and complex in its type since it involves an interplay between diffusion of a chemical wave coupled with a polymorphic transformation of $\mathrm{HgI}_{2}$ along with a redissolution of the precipitate, all occurring in a heterogeneous mixture, in a porous medium (the gel). Moreover, it is obvious in all of our experiments that the propagating front is defined by a colorless outline. This outline is reasoned to be the complex $\mathrm{K}_{2} \mathrm{HgI}_{4}$ formed due to the excess iodide solution.

\subsection{Spatiotemporal patterns}

3.2.1 Effect of concentrations. As depicted in Fig. 1, the traveling wave patterns that we encounter in this system include single circular waves, the so-called targets, spirals, and ripples similar to cardioid structures originating from a double spiral core. The selection of the patterns, their coexistence, and their characteristic wavelengths depends on the initial concentrations of the reactants. They preserve their structure during propagation. If they collide with each other they merge, and eventually lose stability and break up into chaotic patterns. In Fig. 2 we present a phase diagram that provides a qualitative summary of the most probable self-organized patterns to nucleate at each concentration. It is to be noted that all patterns can be suppressed if the gel medium is carefully prepared to eliminate all nucleating sites such as tiny air bubbles. The figure relates different outer concentrations with different inner concentrations showing specifically which pattern has the highest probability to occur at each pair of inner/outer concentration combinations. This informative figure was produced from a large number of experimental repetitions, and an accurate probability of the results was combined to reach this analysis. As the figure shows, target patterns have the highest probability of appearance at low outer concentrations and as concentration increases, the spiral and ripple-like patterns become the more dominant structures to nucleate.

It is noteworthy to state that the nucleating self-organized patterns are highly dependent on the initial mercuric concentration, $\left[\mathrm{Hg}^{2+}\right]_{0}$, in the gel medium. They only appear in a limited range labeled the "excitable region" which exists between $0.16 \mathrm{M}$ and $0.26 \mathrm{M}$ of $\left[\mathrm{Hg}^{2+}\right]_{0}$. This "excitable region" coincides perfectly with our previously obtained results on pattern formation in mercuric iodide chemical waves propagating in a two-dimensional system.

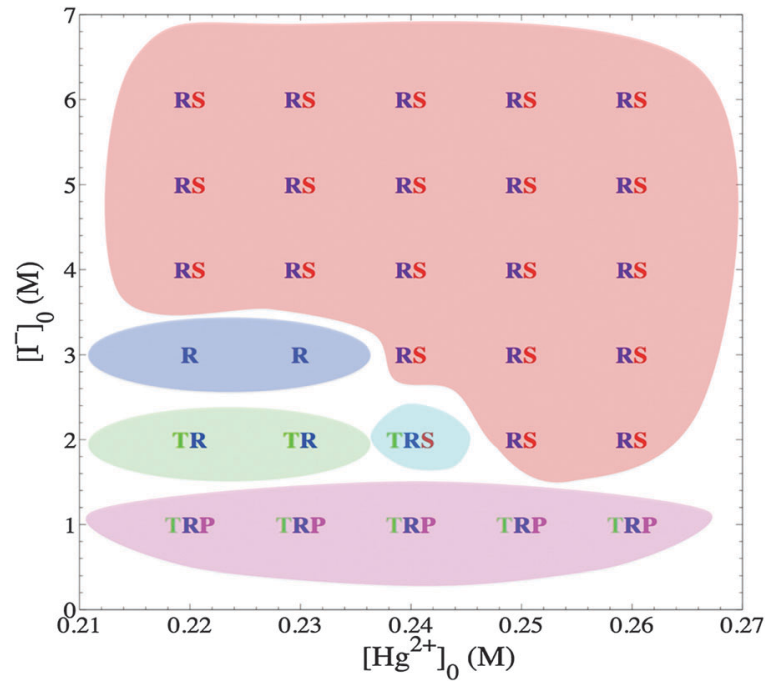

Fig. 2 Phase diagram as a function of the outer $\left[\mathrm{I}^{-}\right]_{0}$ and inner $\left[\mathrm{Hg}^{2+}\right]_{0}$, showing the most probable type of self-organized patterns appearing at every pair of inner/outer concentrations. (P) denotes phase wave, (T) target, (R) ripple, (S) spiral. At least 10 experiments were performed for each pair of concentrations $\left(\left[\mathrm{I}^{-}\right]_{0},\left[\mathrm{Hg}^{2+}\right]_{0}\right)$.

The pattern formation was observed in the same concentration range and no patterns appeared when reaction was carried out outside this range. ${ }^{28}$ This brings upon the fact that there exists a relationship between patterns occurring in the two-dimensional system and the three-dimensional patterns appearing on the surface of the gel. This aspect is very clearly explained in the work of Tinsley et al. ${ }^{29}$ in the work they carried out on a similar system yet precipitating aluminum hydroxide.

On the other hand, the outer electrolyte plays the role of determining the probability of the pattern, which is going to majorly nucleate. As Fig. 2 shows, at high outer values the major patterns are spirals and ripples, yet as we go lower in concentration, targets become the more major pattern to be observed. Another role of the outer electrolyte noticed is the actual quantity of nucleation sites that actually produce a pattern. At an initial iodide concentration, $\left[\mathrm{I}^{-}\right]_{0}=1.0-2.0 \mathrm{M}$, experiments show nucleation of about 40-50 patterns, but when the concentration increased to $3.0 \mathrm{M}$ and above, the count dropped to less than 10 patterns. Therefore, for simply increasing the probability of nucleation we decrease the outer concentration, yet we expect a crowded reactor with small patterns. If we require a larger pattern relative to the size of the reactor with minimal collision with other patterns, we need to increase the outer concentration.

3.2.2 Polymorphic transformation of the pattern background. The sinking precipitation layer (we name the background), which is parallel to the gel surface and orthogonal to the observation direction (top view), travels through the gel medium due to precipitation and complex formation. In the majority of experiments we carry out, when we initialize the reactions, the chemistry of $\mathrm{HgI}_{2}$ determines that the first polymorph to precipitate is the thermodynamically unstable orange form. On this unperturbed area of the precipitate, the red chemical waves nucleate and 
propagate to form the reported patterns. In the meantime, as the patterns are growing, a polymorphic transformation occurs which changes the color of the background from the unstable orange polymorph (Fig. 3A) to the metastable yellow polymorph (Fig. 3B). This yellow persists for a significant period of the reaction and it is the main domain of which the pattern formation is observed. As the reaction and pattern propagation proceed, the thermodynamically stable red polymorph starts nucleating out of the yellow background and starts appearing as red dots (Fig. 3C), which eventually invades the entire reaction surface (Fig. 3D). The rate of this transformation varies depending on the initial concentration of reactants. Similar alternations between the polymorphs of mercuric iodide have been recently reported. ${ }^{27}$ Fig. 3 shows the time evolution of the polymorphic transformation of the background of the patterns, expressing the significant polymorph color at each stage.

3.2.3 Three-dimensional superdiffusive targets. Classical reaction-diffusion equations are able to reproduce the propogation velocities of waves in various excitable and oscillatory systems, which are either constant or diffusive such that their mean-square displacement of the front, $\overline{R^{2}}$, is a linear function of time; i.e. $\overline{R^{2}} \sim t^{2 \alpha}$, where $\alpha=0.5$. This derives from the fact that at the microscopic level, particles undergo Brownian motion whose time increments obey Gaussian statistics. However, exceptions exist in numerous complex systems such as in turbulent fluids, ${ }^{30}$ chaotic dynamics ${ }^{31}$ and disordered media, ${ }^{32}$ whereby anomalous ${ }^{33}$ $\operatorname{sub}-(\alpha<0.5)$ and superdiffusion $(\alpha>0.5)$ mechanisms are the driving forces governing the physics of transport. ${ }^{34}$ While in chemistry subdiffusion is frequently encountered for example when reactions take place in porous media, superdiffusion is only seen in special chemical systems subject to utilization of external forces. ${ }^{31,35,36}$ We study in this section target patterns that exhibit superdiffusive propagation without external interference.

Target patterns are circular waves that arise from small modified regions that can act as pacemakers due to the slightest perturbations such as the presence of dust particles, gas bubbles or surface defects. ${ }^{37}$ In homogenous media, these regions typically have a local oscillation frequency higher than that of uniform oscillations. ${ }^{38}$ On the other hand, the targets in our system nucleate at the air bubbles trapped in the gel after its solidification and lack continuous stimulation. Since in our media such perturbative bubbles are available in moderate amounts, several circular patterns are clearly observed.
When the gel preparation is carefully performed with the elimination of the bubbles, no initiation of targets can be observed.

Moreover, the examination of the targets using scanning electron microscopy (SEM) reveals a three-dimensional ridgy wavefront where the boundary of the circular target is actually higher than its internal part with a range between 70 and $100 \mu \mathrm{m}$ while the gel outside the target is only around $20-40 \mu \mathrm{m}$ lower. ${ }^{19}$

By studying the propagation profiles portraying the spread of our targets in time, ${ }^{19}$ we found that the speeds of these waves are not constant for various inner concentrations and at a constant outer concentration of $1.0 \mathrm{M}$. The radii were measured throughout the time the patterns are stable before the breakup. In addition, the highest inner concentration indicates the fastest pattern breakup, which may be attributed to the highest initial excitability; thereby the pattern loses stability at an earlier stage. We also realize that at this highest concentration a relatively high velocity of wave propagation is achieved. The diffusion curves, for all cases studied, fit very well a time power law equation of the form $R \sim t^{\alpha}$ with exponents $\alpha$ well above 0.5 , which is the expected value in regular diffusive (Fickian) dynamics as these are diffusion-limited reactions. As the inner concentration is increased from $0.20 \mathrm{M}$ to $0.26 \mathrm{M}$, the power $\alpha$ increases as well, and ranges from 0.65 to 0.84 . This confirms that these patterns are not governed by Fickian diffusion, with underlying microscopic transport driven by an uncorrelated, Markovian, Gaussian process, and reaction kinetics as well as the gel matrix may be playing a stronger role in their dynamics pushing transport into the realm of non-Gaussian processes such as Lévy flights. ${ }^{19}$ It is noteworthy that at every given experiment we select at random up to 5 targets and perform the same aforementioned curve fitting, only to obtain the same value of the exponent $\alpha$. Similar measurements are also performed on spirals and targets with different initial outer and inner concentrations and in all these measurements superdiffusive behavior is also achieved. ${ }^{19}$

When two targets collide they do not overlap but actually merge and form a larger yet deformed pattern. This is a result of the depletion of $\alpha-\mathrm{HgI}_{2}$, constituting the chemical wave, from the region of collision, which transforms to an unperturbed quasi-steady $\beta-\mathrm{HgI}_{2}$ (see $\mathrm{ESI} \dagger$ for a more descriptive video of this incident). After prolonged evolution the patterns are condemned to lose their stability leading to their breakup followed by chemical turbulence.

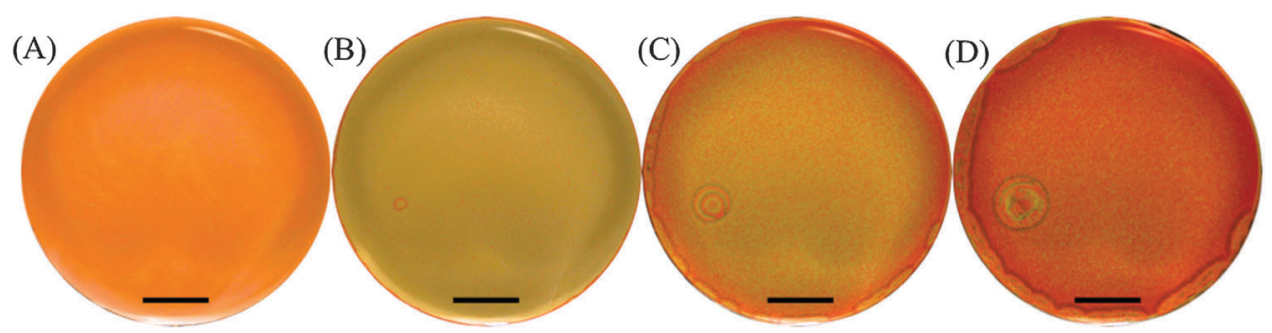

Fig. 3 Evolution of the background of the patterns (unperturbed area of the precipitate) in mercuric iodide with time. Initial conditions: $1 \%$ agar gel; $\left[\mathrm{HgCl}_{2}\right]=0.24 \mathrm{M}$ (inner), $[\mathrm{KI}]=3.0 \mathrm{M}$ (outer). Time after initialization: (A) $10 \mathrm{~s}$ (orange) (B) $125 \mathrm{~s}$ (yellow) (C) $430 \mathrm{~s}$ (transition to red) (D) $705 \mathrm{~s}$ (red). The scale bar represents $1.0 \mathrm{~cm}$. 
To add to the characterization of the targets, we also measured the width of the propagating wave and it was found to be fairly constant throughout the reaction. The values are found in the range between 120 and $240 \mu \mathrm{m}$. The width does not show any significant change with time but it is confirmed to slightly decrease as $\left[\mathrm{Hg}^{2+}\right]_{0}$ increases. This fact shares similarity with the BZ reaction in the experimental and theoretical studies conducted by Bugrim et al. ${ }^{39}$ and by Pagola and Vidal, ${ }^{40}$ where it is concluded that the width of the targets remains constant during propagation.

3.2.4 Spirals and ripples. Next rather interesting pattern common to our system is the rotating spiral wave. The interest in this pattern is attributed to its universal character: spiral waves are observed in media with spontaneous oscillations as well ${ }^{41,42}$ and, from a topological point of view, are equivalent ${ }^{43}$ to dislocation type defects in striped patterns like convective rolls. ${ }^{44}$ In this system, as shown in Fig. 1(B), spiral waves rotate steadily about fixed centers with their tip pinned at the nucleation site and their curvature remains constant until the spiral loses stability and breaks up. ${ }^{45}$

Another type of pattern we encountered is the double spiral wave that we call a ripple and is shaped like a cardioid (Fig. 1(C)). It has a distinct feature that it first gets created as a double spiral wave with two inwardly rotating tips, which meander until they meet, and subsequently merge, resulting in a structure that resembles a deformed target pattern. However, contrary to the case of targets, the presence of a seemingly continuous stimulation gives rise to a family of merging double spirals from the same nucleation site producing a wavetrain of cardioid patterns or ripples. The velocities and separation of the successive waves are calculated and found to be unequal. The relationship between the velocities of these wavetrains and their periods can be accounted for by the anomalous dispersion relation $^{20,21}$ as will be shown later on.

The wavelengths of spirals are then measured. For the same pair of inner/outer concentrations and at different propagation times, up to 10 different ripples/spirals are randomly selected from the total domain to measure their wavelengths. The measured wavelengths at a given time for these different patterns are close to each other due to little dispersion. Fig. 4 provides plots representing the change of the average wavelength, computed from the aforementioned selected patterns, with respect to the tested $\left[\mathrm{Hg}^{2+}\right]_{0}$ range at a constant $\left[\mathrm{I}^{-}\right]_{0}$. The wavelength exhibits an inverse relation with the value of reacting concentration. All four plots show that from the lowest to the highest concentration tested, the values of wavelengths are halved. On the other hand, the alteration of $\left[\mathrm{I}^{-}\right]_{0}$, at constant $\left[\mathrm{Hg}^{2+}\right]_{0}$, has the opposite effect on the wavelength, as shown in Fig. 5 . The highest $\left[\mathrm{I}^{-}\right]_{0}$ has a wavelength measurement of nearly double that of the lowest $\left[\mathrm{I}^{-}\right]_{0}$. The values of wavelengths vary between 0.26 and $1.37 \mathrm{~mm}$, in the figures reported. Plesser et al. performed similar tests and they also reported the decrease of spiral wavelength with the increase of proton concentration in the measurements conducted on the $\mathrm{BZ}$ reaction patterns. ${ }^{46}$

For a given ripple pattern, the distance covered over time, from the nucleation site, of three consecutive waves starting at the moment each one appeared is shown in Fig. 6. This figure

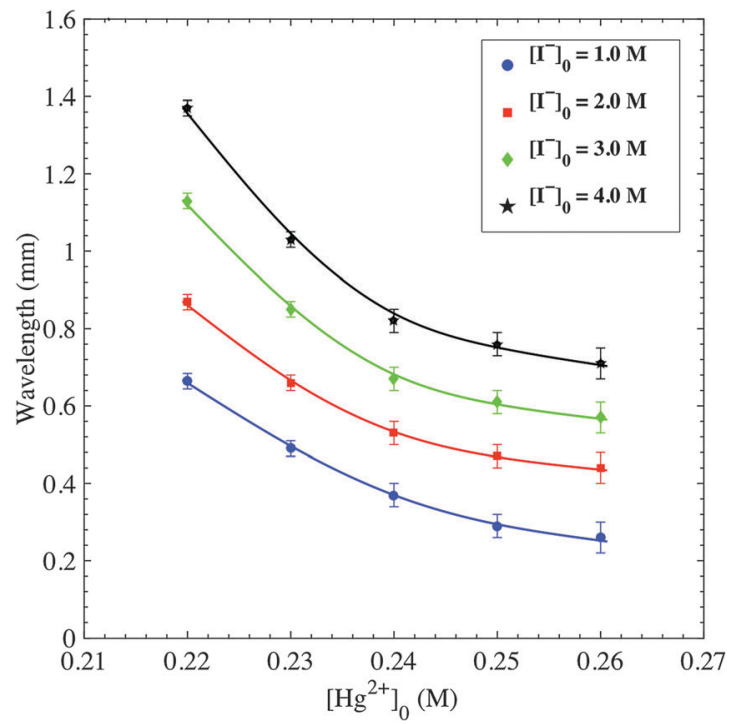

Fig. 4 Plots of wavelengths of spiral and ripple patterns versus inner $\left[\mathrm{Hg}^{2+}\right]_{0}$. Initial conditions: $1 \%$ agar gel, inner $\left[\mathrm{Hg}^{2+}\right]_{0}=0.22-0.26 \mathrm{M}$, $\left[\mathrm{I}^{-}\right]_{0}=1.0-4.0 \mathrm{M}$.

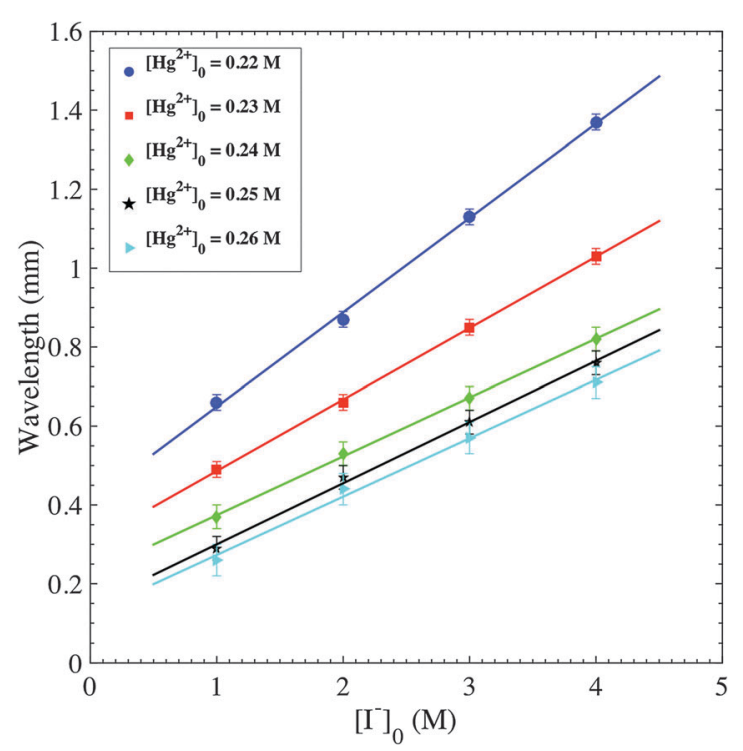

Fig. 5 Plots of wavelengths of spiral and ripple patterns versus outer $\left[\mathrm{II}^{-}\right]_{0}$. Initial conditions: $1 \%$ agar gel, inner $\left[\mathrm{Hg}^{2+}\right]_{0}=0.22-0.26 \mathrm{M},\left[\mathrm{I}^{-}\right]_{0}=$ 1.0-4.0 M. Plots were fitted to a linear equation relating wavelength as a function of $\left[\mathrm{I}^{-}\right]_{0} . \beta$ (circle) $=0.24 \pm 0.03, \beta$ (square) $=0.18 \pm 0.01, \beta$ $($ diamond $)=0.15 \pm 0.01, \beta$ (star) $=0.16 \pm 0.03, \beta$ (triangle $)=0.15 \pm 0.03$.

uncovers that the leading wave propagates at a relatively slow velocity than the following waves. As shown, the velocities are relatively different, and at an indicated time the leading wave slows down as the second wave catches up and gradually stacks until they merge thus forming one large front. The "attractive" transition between wave 1 and 2 is clearly visible on the graph and is typical of ripples dynamics in this system. Such merging occurs when waves are separated by about $1 \mathrm{~mm}$. The right part of Fig. 6 shows the evolution of the ripple structure that is analyzed in the plots on the left of the same figure and clarifies 


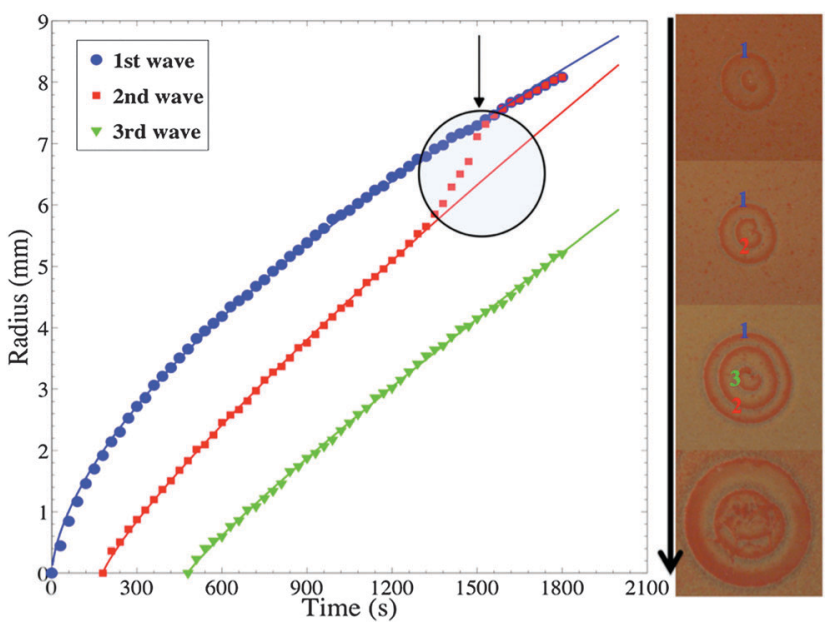

Fig. 6 Plots of the radius traveled by the wavetrain of ripples, shown in the panels on the right-hand side of the graph, from the nucleation site versus time. The plots of three consecutive waves and their relative speeds are represented. Initial conditions: $1 \%$ agar gel, inner $\left[\mathrm{Hg}^{2+}\right]_{0}=0.23 \mathrm{M}$, $\left[\mathrm{I}^{-}\right]_{0}=4.0 \mathrm{M}$. Plots were fitted to a power equation relating radius $(\text { time })^{\mu} . \mu$ (circle) $=0.62 \pm 0.01, \mu$ (square) $=0.84 \pm 0.01, \mu$ (triangle) $=$ $0.90 \pm 0.01$. The arrow indicates the time at which the collision between wave 1 and 2 occurs. The circle indicates the transition dynamics when waves are about $1 \mathrm{~mm}$ close to each other.

the described three waves and how they collide with each other leading to the breakup of the pattern. The plots in Fig. 6 also fit a time power law $\left(R \sim t^{\alpha}\right)$, with exponent values all significantly above 0.5 , clearly indicating superdiffusive dynamics. The first wave, which is the slowest, has a power value of 0.62 followed by the second wave with a power of 0.84 . This explains the reason why the second wave catches up to the leading wave fairly quickly. The power value of the third wave is close to 0.9. This merging of propagating waves indicates that this heterogeneous system exhibits remarkable similarities with excitable systems due to anomalous dispersion (see Fig. 3c by Steinbock et $\left.a l^{20}\right)$. For such wave merging, the dispersion curves plotted in Fig. 6 start at a high value of wave velocity and do not attain a fixed velocity at a fixed wavelength. The dispersion curves clearly have negative slopes $(\mathrm{d} c / \mathrm{d} T)$ down to very small periods and the velocity is always greater than the characteristic value of a solitary wave $\left(c_{0}=0.0036 \mathrm{~mm} \mathrm{~s}^{-1}\right)$ with a maximum starting velocity that is about 6 times this value (Fig. 7).

3.2.5 Phase-like wave. In our experiments, we also detected a fourth interesting pattern that only persists for a short period of time of about 40-50 seconds. This pattern was given the name "phase-like wave" because it looked like a firing wave, which appeared all over the surface of the gel and consisted of the "firing" of the orange polymorph on a homogeneous domain of the yellow polymorph of $\mathrm{HgI}_{2}$. Fig. 8 shows snapshots of the evolution of a phase wave with time. The significance of this pattern, which emerges for a time interval ranging from 1 second to about 3 seconds, is that it is does not appear on a certain nucleation site, but it invades the whole area of the reactor forming a large spiral wave which eventually breaks down and completely disappears and the system returns to the initial yellow domain.

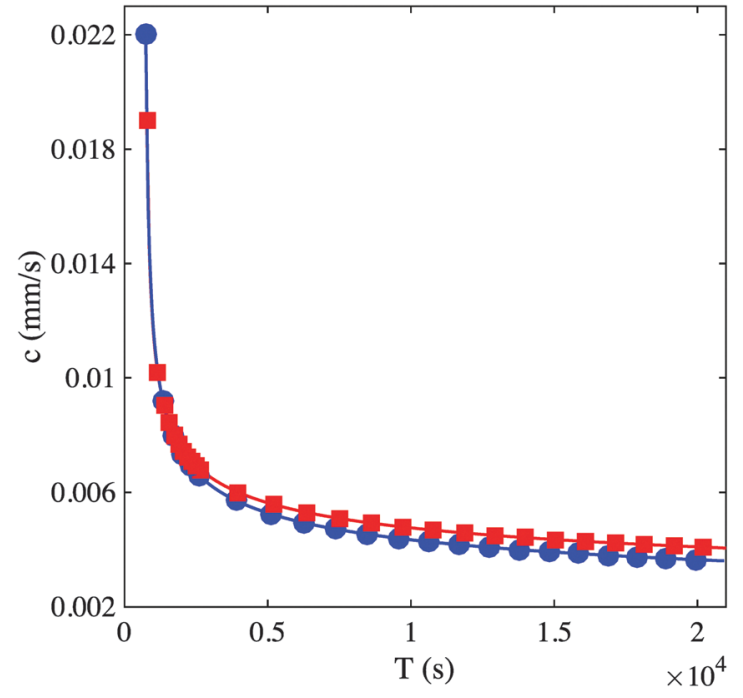

Fig. 7 Plots of the dispersion relations of waves in Fig. 6. Square and circles are data plots obtained from the wave pairs $(1,2)$ and $(2,3)$, respectively. The speed $c$ is computed by time differentiation of the nonlinear fits to the curves in Fig. 6 . The period $T$ is computed as the difference between the passage times of subsequent waves through a given position in the medium. The overlap of the data points from the two pairs is indicative of consistency of the results.

This type of non-propagating or "breathing" domain has been reported in excitable media ${ }^{45,47-49}$ and occurs when the diffusion length of the slow variable exceeds that of the fast variable and consequently diffusion of the slow variable ahead of the excited domain can inhibit further excitation and, at the same time, balance the local production of the fast variable, thus averting the recovery to the rest state. This might be followed by a Hopf bifurcation, leading to the "breathing" behavior that expands and shrinks periodically in time. After this "firing" pattern subsides, the system might exhibit nucleation of targets, ripples and spirals.

In addition, this pattern is only observed right after initialization and specifically has the highest probability of occurrence at an outer iodide concentration of $1.0 \mathrm{M}$. There are rare cases where this pattern is observed at an iodide concentration of $2.0 \mathrm{M}$, but is definitely nonexistent at a concentration of $3.0 \mathrm{M}$ and above.

\subsection{Pattern breakup}

Transition from regular patterns to spatiotemporal chaos is encountered in various systems such as the reaction-diffusion system, ${ }^{12,50,51}$ fluid flows, ${ }^{52,53}$ cardiac tissue, ${ }^{6,54}$ and bacterial colonies. ${ }^{55}$ In particular, spiral wave instability in a reactiondiffusion system leading to transition to a state of defectmediated turbulence is subject to extensive investigations. ${ }^{56,57}$

In this work, the dynamics of the breakup of the targets and spirals/ripples, depicted in Fig. 9, is also investigated. Although the breakup mechanisms in the two cases are different, ultimately the system falls into a state of defect-mediated turbulence in both cases.

In the case of targets (Fig. 9(A-C)), the breakup is initiated as defects at different locations on the boundary of the wave itself are formed. These defects propagate inwards towards the 
(A)

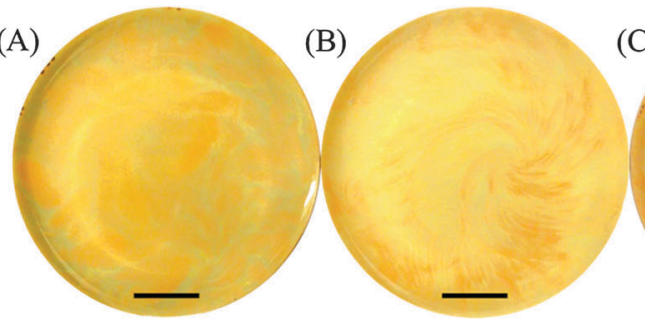

(C)

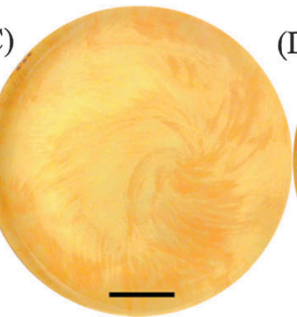

(D)

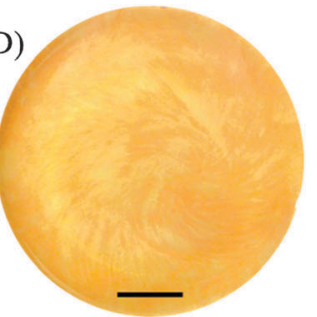

Fig. 8 Evolution of the phase-like wave in $\mathrm{Hgl}_{2}$ with time (top view). Initial conditions: $1 \%$ agar gel, inner $\left[\mathrm{Hg}^{2+}\right]_{0}=0.23 \mathrm{M}$ outer $\left[\mathrm{I}^{-}\right]_{0}=1.0 \mathrm{M}$. (A) $5 \mathrm{~s}(\mathrm{~B})$ $15 \mathrm{~s}$ (C) $25 \mathrm{~s}$ (D) $35 \mathrm{~s}$, after initialization. The scale bar represents $1 \mathrm{~cm}$.

nucleation center of the pattern, leaving behind a state of chemical turbulence (see ESI $\dagger$ ). The cause of these defects is not very well understood yet. However, bubbles, which do not previously nucleate, may sometimes act as destabilizing obstacles. Furthermore, the higher the $\left[\mathrm{Hg}^{2+}\right]_{0}$ or $\left[\mathrm{I}^{-}\right]_{0}$ are, the earlier the onset of the instability takes place and the smaller the radius is before the target collapses.
In the case of spirals (Fig. 9(D-F)) and ripples (Fig. 9(G-I)), the breaking up is initiated at the center of the pattern and propagates outwards to form a cascade of birth of smaller spirals leading eventually to chemical turbulence. This dynamical cascade that leads to turbulence seems to be also a consequence of an anomalous dispersion, which is clear in the case of Fig. $9(\mathrm{G}-\mathrm{I})$ whereby the outermost circular fronts of two ripples collide and

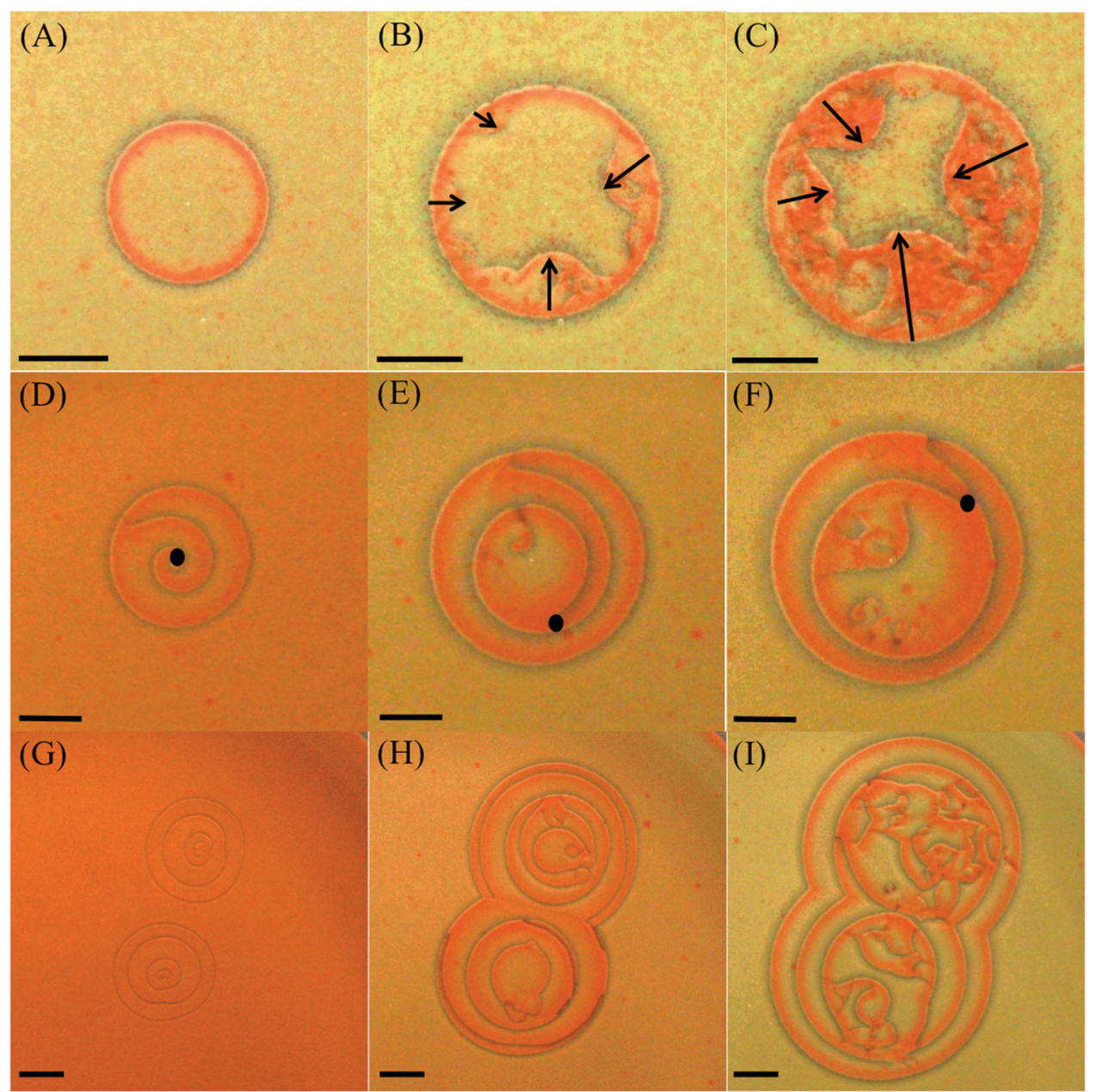

Fig. 9 Time evolution of the propagating wave patterns until their breakup. Initial conditions: $1 \%$ agar. Target breakup: $\left[\mathrm{Hg}^{2+}\right]_{0}=0.26 \mathrm{M}\left[\mathrm{I}^{-}\right]_{0}=3.0 \mathrm{M}$; (A) $230 \mathrm{~s}$ (B) $395 \mathrm{~s}$ (C) $535 \mathrm{~s}$; arrows indicate the direction of propagation of the defects. Spiral breakup: $\left[\mathrm{Hg}^{2+}\right]_{0}=0.23 \mathrm{M},\left[\mathrm{I}^{-}\right]_{0}=4.0 \mathrm{M}$; (D) $180 \mathrm{~s}$ (E) $345 \mathrm{~s}$ (F) $445 \mathrm{~s}$; the black dots indicate the location of the tip of the spiral. Ripple breakup: $\left[\mathrm{Hg}^{2+}\right]_{0}=0.24 \mathrm{M}$, $\left[\mathrm{Il}^{-}\right]_{0}=5.0 \mathrm{M}$; (G) $195 \mathrm{~s}(\mathrm{H}) 425 \mathrm{~s}$ (I) $680 \mathrm{~s}$. What is also interesting in this last series of snapshots is the merging of two ripples before destruction. Times reported represent the period after initialization. The scale bar represents $0.25 \mathrm{~cm}$. 
mutually annihilate forming an 8-shaped leading front. The same thing happens with the second fronts emitted by those ripples but this new front has a higher velocity than the former, leading to a front-to-back collision. As the third and fastest waves arrive and collide with the outermost already merged fronts they create several perturbation sites that act as new pacemakers with higher frequencies than the two ripples. However, these pacemakers fail to produce any organized pattern and eventually lead to chemical turbulence.

In the case of the spiral in Fig. 9(D-F), the transition to turbulence is akin to the instability encountered in spirals, which is caused by the Doppler effect encountered in the BZ reaction. ${ }^{51}$ This instability is initiated by a Hopf bifurcation acting on the spiral core, which results eventually in large meandering of the spiral tip towards its adjacent wave, causing it to break up and produce more than one perturbation site. The spiral tip snaps off the pinning site and start meandering as shown in Fig. 9(D-F) where the black dots indicates the location of the tip. The new perturbation sites also nucleate spirals with a higher frequency than the initial spiral due to the anomalous dispersive dynamics, which also meander causing more chaos. The new defects introduce more defects, which in their turn contribute more to the pattern chaos spawning a saturation of deformity and ultimately producing a state of chemical turbulence. ${ }^{58}$ This transition to turbulence is accelerated by the increase of the control parameter $\left[\mathrm{Hg}^{2+}\right]_{0}$. The Doppler effect also induces a continuous alteration of the local wavelength of the spiral or the ripple before breakup, as shown in Fig. 9(D-I).

There also exists in our system a second route to pattern break up and that is specific to spirals and ripples. As their structures show, they are made of several propagating waves, arising from the initial nucleation site. In ripples, as the leading wave diffuses with time it slows down allowing the wave following it to catch up and collide with it. This collision produces a larger diffusing front and in many cases causes the pattern to lose stability and break up into a chaotic pattern. It is noteworthy that the total number of waves fired in the case of ripples before breakup increases as the initial concentration of the outer electrolyte $\left[\mathrm{I}^{-}\right]_{0}$ increases. For example, in the case of $\left[\mathrm{I}^{-}\right]_{0}$ less than or equal to $4.0 \mathrm{M}$, not more than 3 waves can be fired by a single ripple, whereas in the case of $\left[\mathrm{I}^{-}\right]_{0}$ greater than or equal to $5.0 \mathrm{M}$, one can easily distinguish more than 5 propagating waves for each ripple before breakup. Similarly in spirals, the inner turns become faster than the outermost turn, thus deforming the structure as it evolves. When obvious spatially altered waves emerge, the distance in space between consecutive waves varies. As the $\left[\mathrm{Hg}^{2+}\right]_{0}$ increases, the amount of alterations increases thus leading to this kind of breakup. ${ }^{58}$

\section{Conclusion}

We report in this study an interesting collection of self-organized patterns observed for the first time in a heterogeneous system that consists of a reaction-diffusion system involving the precipitation of $\mathrm{HgI}_{2}$. The patterns appearing include targets, spirals and ripples that undergo $\mathrm{HgI}_{2}$ polymorphic transformation and also experience instabilities leading to chemical turbulence. The dispersion relation relating the speed of the waves to their wavelengths seems to follow the anomalous trend. We also show that the dynamics of the propagating waves is superdiffusive and this fact constitutes the first example of this kind in chemistry without the utilization of external forces. This will definitely open the door for further investigations including the theoretical understanding of this anomalous behavior and the formation of these patterns, leading to predictive models. In addition, the main concern and a future endeavor is to apply external forces onto our patterns, such as thermal, chemical, electrical, etc. alterations, ${ }^{59,60}$ to reduce or suppress pattern instabilities and chemical turbulence.

\section{Conflicts of interest}

The authors declare no competing financial interests.

\section{Acknowledgements}

This work was supported by the Lebanese Council for National Scientific Research (LCNSR), the University Research Board, American University of Beirut and the Hungarian Research Fund (OTKA K104666).

\section{References}

1 B. Grzybowski, Chemistry in Motion: Reaction-diffusion Systems for Micro-and Nanotechnology, Wiley Online Library, 2009.

2 B. A. Grzybowski, K. J. Bishop, C. J. Campbell, M. Fialkowski and S. K. Smoukov, Soft Matter, 2005, 1, 114-128.

3 M. C. Cross and P. C. Hohenberg, Rev. Mod. Phys., 1993, 65, 851.

4 A. Panfilov, R. Keldermann and M. Nash, Proc. Natl. Acad. Sci. U. S. A., 2007, 104, 7922-7926.

5 M. Loose, E. Fischer-Friedrich, J. Ries, K. Kruse and P. Schwille, Science, 2008, 320, 789-792.

6 L. D. Weise and A. V. Panfilov, PLoS One, 2011, 6, e27264.

7 S. C. Müller and J. Ross, J. Phys. Chem. A, 2003, 107, 7997-8008.

8 P. Hantz, J. Phys. Chem. B, 2000, 104, 4266-4272.

9 T. Narita and M. Tokita, Langmuir, 2006, 22, 349-352.

10 P. De Kepper, I. R. Epstein and K. Kustin, J. Am. Chem. Soc., 1981, 103, 2133-2134.

11 A. Zaikin and A. Zhabotinsky, Nature, 1970, 225, 535-537.

12 Q. Ouyang and H. L. Swinney, Nature, 1991, 352, 610-612.

13 A. M. Turing, Bull. Math. Biol., 1990, 52, 153-197.

14 R. Imbihl and G. Ertl, Chem. Rev., 1995, 95, 697-733.

15 S. Jakubith, H. Rotermund, W. Engel, A. Von Oertzen and G. Ertl, Phys. Rev. Lett., 1990, 65, 3013-3016.

16 A. Volford, F. Izsák, M. Ripszám and I. Lagzi, Langmuir, 2007, 23, 961-964.

17 G. Fernández-García and V. Pérez-Muñuzuri, Eur. Phys. J.: Spec. Top., 2008, 165, 169-174.

18 P. M. Wood and J. Ross, J. Chem. Phys., 1985, 82, 1924. 
19 M. M. Ayass, I. Lagzi and M. Al-Ghoul, Phys. Chem. Chem. Phys., 2014, 16, 24656-24660.

20 C. T. Hamik, N. Manz and O. Steinbock, J. Phys. Chem. A, 2001, 105, 6144-6153.

21 N. Manz, C. Hamik and O. Steinbock, Phys. Rev. Lett., 2004, 92, 248301.

22 M. M. Ayass, A. Abi Mansour and M. Al-Ghoul, J. Phys. Chem. A, 2014, 118, 7725-7731.

23 G. A. Jeffrey and M. Vlasse, Inorg. Chem., 1967, 6, 396-399.

24 M. Hostettler, H. Birkedal and D. Schwarzenbach, Acta Crystallogr., Sect. B: Struct. Sci., 2002, 58, 903-913.

25 M. Hostettler, H. Birkedal and D. Schwarzenbach, Helv. Chim. Acta, 2003, 86, 1410-1422.

26 I. Das, A. Pushkarna and N. R. Agrawal, J. Phys. Chem., 1989, 93, 7269-7275.

27 R. J. Field and R. M. Noyes, J. Am. Chem. Soc., 1974, 96, 2001-2006.

28 M. M. Ayass and M. Al-Ghoul, J. Phys. Chem. A, 2014, 118, 3857-3865.

29 M. R. Tinsley, D. Collison and K. Showalter, J. Phys. Chem. A, 2013, 117, 12719-12725.

30 G. Boffetta, F. De Lillo and S. Musacchio, Phys. Rev. E: Stat., Nonlinear, Soft Matter Phys., 2012, 85, 066322.

31 M. Paoletti, C. Nugent and T. Solomon, Phys. Rev. Lett., 2006, 96, 124101.

32 J.-P. Bouchaud and A. Georges, Phys. Rep., 1990, 195, 127-293.

33 R. Klages, G. Radons and I. M. Sokolov, Anomalous Transport, Wiley. com, 2008.

34 V. Shkilev, J. Exp. Theor. Phys, 2008, 107, 892-898.

35 Z. Noszticzius, Z. Bodnar, L. Garamszegi and M. Wittmann, J. Phys. Chem., 1991, 95, 6575-6580.

36 A. von Kameke, F. Huhn, G. Fernández-García, A. Muñuzuri and V. Pérez-Muñuzuri, Phys. Rev. E: Stat., Nonlinear, Soft Matter Phys., 2010, 81, 066211.

37 A. S. Mikhailov and K. Showalter, Phys. Rep., 2006, 425, 79-194.

38 Y. Kuramoto, Chemical Oscillations, Waves, and Turbulence, Courier Dover Publications, 2003.
39 A. E. Bugrim, M. Dolnik, A. M. Zhabotinsky and I. R. Epstein, J. Phys. Chem., 1996, 100, 19017-19022.

40 A. Pagola and C. Vidal, J. Phys. Chem., 1987, 91, 501-503.

41 P. S. Hagan, SIAM J. Appl. Math., 1982, 42, 762-786.

42 J. Ma, B. Hu, C. Wang and W. Jin, Nonlinear Dyn., 2013, 1-11.

43 Th. Walter, W. Pesch and E. Bodenschatz, Chaos, 2004, 14, 933-939.

44 E. D. Siggia and A. Zippelius, Phys. Rev. A: At., Mol., Opt. Phys., 1981, 24, 1036.

45 E. Meron, Phys. Rep., 1992, 218, 1-66.

46 T. Plesser, S. C. Mueller and B. Hess, J. Phys. Chem., 1990, 94, 7501-7507.

47 G. Ermentrout, S. Hastings and W. Troy, SIAMJ. Appl. Math., 1984, 44, 1133-1149.

48 Y. Kuramoto, Prog. Theor. Phys., 1980, 63, 1885-1903.

49 Y. Nishiura and M. Mimura, SIAM J. Appl. Math., 1989, 49, 481-514.

50 A. Belmonte, J.-M. Flesselles and Q. Ouyang, EPL, 1996, 35, 665 .

51 Q. Ouyang, H. L. Swinney and G. Li, Phys. Rev. Lett., 2000, 84, 1047-1050.

52 R. E. Ecke, Y. Hu, R. Mainieri and G. Ahlers, Science, 1995, 269, 1704-1707.

53 N. Mukolobwiez, A. Chiffaudel and F. Daviaud, Phys. Rev. Lett., 1998, 80, 4661.

54 R. F. Gilmour, Drug Discovery Today, 2003, 8, 162-167.

55 K. J. Lee, E. C. Cox and R. E. Goldstein, Phys. Rev. Lett., 1996, 76, 1174.

56 B.-W. Li, L.-Y. Deng and H. Zhang, Phys. Rev. E: Stat., Nonlinear, Soft Matter Phys., 2013, 87, 042905.

57 O. Steinbock and H. Engel, Eng. Polym. Chem. Complexity, 2013, 11, 147.

58 L. Q. Zhou and Q. Ouyang, J. Phys. Chem. A, 2001, 105, 112-118.

59 Q. Lou, J.-X. Chen, Y.-H. Zhao, F.-R. Shen, Y. Fu, L.-L. Wang and Y. Liu, Phys. Rev. E: Stat., Nonlinear, Soft Matter Phys., 2012, 85, 026213.

60 P. Kaštánek, J. Kosek, D. Šnita, I. Schreiber and M. Marek, Phys. D, 1995, 84, 79-94. 